\title{
Les formations en communication à l'épreuve de la demande sociale
}

Fathallah Daghmi

\section{(2) OpenEdition}

1 Journals

Édition électronique

URL : http://journals.openedition.org/communicationorganisation/3487

DOI : 10.4000/communicationorganisation.3487

ISSN : 1775-3546

Éditeur

Presses universitaires de Bordeaux

Édition imprimée

Date de publication : 1 octobre 2006

Pagination : 250-264

ISSN : 1168-5549

Référence électronique

Fathallah Daghmi, «Les formations en communication à l'épreuve de la demande sociale »,

Communication et organisation [En ligne], 30 | 2006, mis en ligne le 21 juin 2012, consulté le 19 avril

2019. URL : http://journals.openedition.org/communicationorganisation/3487 ; DOI : 10.4000/

communicationorganisation.3487

Ce document a été généré automatiquement le 19 avril 2019.

(c) Presses universitaires de Bordeaux 


\title{
Les formations en communication à l'épreuve de la demande sociale
}

\author{
Fathallah Daghmi
}

1 L'implantation à l'université des Antilles et de la Guyane (UAG), voilà cinq ans, d'une formation à la communication répond initialement à un objectif précis. Il s'agit de la volonté d'accueillir sur place les étudiants des Départements français d'Amérique- DFA (Martinique, Guadeloupe et Guyane) désireux d'entreprendre des études dans ce domaine ${ }^{1}$. Dans ces régions, les métiers de la communication sont présents dans plusieurs secteurs : la presse et les médias audiovisuels, la communication des organisations (au niveau des trois régions, des trois départements, des municipalités ainsi que des entreprises). Elle existe sous toutes ses formes classiques, un grand nombre d'organisations et d'associations qui ont pour objectif la formation, la diffusion de la science et de l'éducation.

2 Par ailleurs les ambitions de ces régions, face au développement de la «société de l'information"sont importantes, comme en témoignent la place prise par les TIC. L'objectif de la formation ${ }^{2}$ est, entre autres, de permettre au niveau local aux étudiants, qui jusque là étaient contraints de quitter les DFA, de se former en communication ${ }^{3}$. Les compétences recherchées concernent la communication des organisations ${ }^{4}$ et les théories de l'information et de la communication permettant d'analyser des études de cas et de les référer à des concepts clairs.

3 Ainsi, le savoir-faire acquis, lors de la formation permet de répondre aux exigences professionnelles. Ces dernières, il faut le reconnaître, sont souvent liées à la réalisation de supports de communication. Ces outils sont conçus et réalisés soit après analyse et réflexion (mémoire) soit, sans réelle analyse, en réponse à une demande urgente des organisations ${ }^{5}$.

4 En définitive, les futurs chargés de communication doivent répondre à deux exigences : universitaire et professionnelle/marché. L'exigence universitaire est déterminée en grande partie par l'application des connaissances théoriques et pratiques et méthodes d'analyse et de réflexion relatives, entres autres, aux Sciences de l'information et de la 
communication (Sic) 6 . A travers des actions de communication, les étudiants tentent de construire des objets de recherches qui correspondent aux approches et points de vue de Sic. Cette perspective considère la communication comme étant fondamentalement technique au sens où elle est une mise en œuvre de savoirs et de connaissances scientifiques et de savoir-faire technique afin de produire des objets: supports, dispositifs, règles et normes, situations, etc. (Davallon, 2004, 36).

Concrètement, les étudiants en communication mettent en application leurs connaissances par l'analyse d'un aspect, d'un phénomène ou d'un problème lié à la communication organisationnelle. Le résultat se déploie par une méthodologie et un travail de réflexion charpenté par un cadrage théorique et consolidé par la mise en œuvre d'outils et méthodes de recueil et traitement de données préconisées par les Sic.Dans ce sens, de quelle façon la formation en communication répond-elle à la demande sociale des organisations dans les DFA?

6 On s'interroge principalement dans ce cadre sur la confrontation des étudiants en communication au terrain des organisations dans les $\mathrm{DFA}^{7}$. Comment réagissent-ils ? Qu'offrent-ils en termes de compétences, de connaissance de la communication et modèles des Sic? Quels angles de traitement proposent-ils aux sujets traités? Et enfin quelles solutions apportent-ils aux organisations afin d'améliorer leur communication?

7 Cette étude de la formation en communication va au-delà d'une présentation spécifique aux DFA. Il s'agit de voir comment les étudiants se saisissent du réel et trouvent des réponses satisfaisantes du point de vue universitaire et professionnel. Le but poursuivi n'est pas de brosser un tableau exhaustif de tous les courants de recherche dans les domaines des Sic et de la sociologie des organisations. Il ne vise pas non plus l'exposition des théories et modèles de communication enseignés dans les DFA. On propose d'identifier les modèles auxquels les étudiants en communication font appel pour approcher, comprendre et répondre à la demande sociale.

\section{La formation en communication face aux organisations}

8 Il existe un décalage incontestable entre, d'une part, la demande exprimée par les organisations et, d'autre part, le besoin effectivement repéré par les étudiants en communication. La première découle globalement d'une vision managériale et utilitaire de la communication. Elle se cantonne souvent dans des besoins liés à la réalisation de supports et d'outils. La seconde, en revanche, est soucieuse de répondre aux besoins de la formation en communication (approche souvent critique). Elle révèle des aspirations qui dépassent la multiplication des supports d'information et de communication et met l'accent sur des besoins d'ordre stratégique qui concernent l'insuffisance ou la non prise en compte du facteur de communication dans la politique globale de l'organisation.

9 Cette remarque est révélatrice de l'image de la fonction de communication et des ses attributions qui restent assez ambiguës dans une bonne partie des organisations dans les DFA. Toutefois, les étudiants en communication tentent de dépasser cet obstacle et proposent une méthodologie et des pistes de recherche qui s'efforcent de répondre à la demande sociale des organisations. 


\section{Une perception réduite de la communication}

10 Les organisations dans les DFA arrivent, tant bien que mal, à reconnaître le rôle que pourrait jouer la communication pour leur fonctionnement. Mais l'intérêt de cette dernière est limité, principalement car elle reste une notion mal définie ou mal appréciée dans l'esprit des dirigeants et salariés. En effet, dans les entreprises privées étudiées, la communication est considérée comme une « sous-discipline » à laquelle on alloue rarement les moyens humains et financiers nécessaires à un fonctionnement optimal.

11 Somme toute, le rôle de la communication dépend des niveaux hiérarchiques. Mais contrairement à ce qu'on pourrait croire, il est minimisé ou « diabolisé » dans les plus hautes fonctions notamment dans certaines collectivités locales. Dans les organisations qui ne disposent pas d'un service de communication, on ne maîtrise pas ce champ et de surcroît l'importance de mettre en place un service dédié à cet effet. Quant aux structures disposant d'un tel service, on ignore ses compétences et son pouvoir. Par conséquent, l'embauche $e^{8}$ du personnel affecté à la communication se fait sur des critères particuliers de formation et les actions réalisées manquent de réflexion, d'approfondissement et ne sont guère insérées dans une stratégie globale de l'organisation.

Dans certaines collectivités, le choix dépend de l'apport en terme d'image et maîtrise du lien avec le monde extérieur, notamment des médias. Ce contexte est donc marqué par une cristallisation des attentes autour d'intérêts politiques, qui se ressentent lors du choix des candidats à l'embauche (par exemple, un journaliste est souvent recruté comme chargé de communication). Concrètement, le service de communication manque de moyen d'action au niveau administratif/juridique. L'absence de stratégie et d'objectifs clairement définis endigue son action et le confine parfois à un aspect « festif ».

Rares sont les expériences qui montrent une réelle prise de conscience quant au rôle fondamental de la communication dans l'organisation. Aussi bien en interne qu'en externe, on s'appuie fortement sur les responsables de communication pour résoudre des problèmes liés au fonctionnement, à la cohésion sociale, à l'image en externe, etc.

\section{Des demandes «techniques »}

14 Les commandes ou demandes des organisations vis-à-vis d'un chargé de communication sont souvent de deux ordres. La première grande tendance concerne la mise en place, l'élaboration ou la mise à jour de supports de communication. La fonction de l'étudiant en communication est avant tout liée à des pratiques concrètes de réalisation d'outils. Elle implique la collecte des informations et leur mise en forme dans des outils: lettres d'information, brochures, journaux, sites web, etc.

La deuxième tendance, minoritaire, est liée à des attentes plus "stratégiques ». En effet, les responsables des organisations espèrent se faire connaître, dynamiser leurs activités ou résoudre des problèmes d'image, auprès du personnel, d'un service ou du fonctionnement global de leurs structures. Dès lors, le futur chargé de communication se voit confier la tâche d'analyser des supports de communication existants en interne ou en externe, de faire le bilan et de mesurer les retombées des outils et activités du service de communication. Parfois, la demande concerne également l'audit de la communication interne de l'organisation. 
16 Par ailleurs, certaines organisations n'ont pas de demande a priori ou de propositions de départ. L'étudiant en communication doit dès lors faire des propositions, après immersion dans la structure, qui vont dans le sens de l'amélioration de la communication interne (objectifs fixés par la formation).

\section{Diversité des problématiques de recherche}

Lorsqu'on fait une analyse des problématiques de recherche développées dans les mémoires de stage des étudiants en communication, on se rend rapidement compte de l'hétérogénéité et de la richesse des pistes d'analyses proposées. Par conséquent, l'extrême largeur du champ d'interrogation du réel montre la diversité des méthodes de recherches des Sic et des demandes sociales des organisations. Globalement, il n'existe pas de différences majeures entre les demandes d'institutions publiques et privées. Cependant, le niveau d'ouverture ainsi que les pratiques et systèmes de communication sont divergents. Plus d'un quart des institutions concernées ne sont pas dotées d'un service de communication et la quasi-totalité des organisations disposent d'une ou deux personnes pour assurer cette fonction. Cela rappelle la jeunesse de l'intérêt pour la communication des organisations dans les DFA.

Les pistes explorées sont multiples9. Tout d'abord, dans les institutions qui ont fait le pari de l'intégration des responsables et moyens de communication, elles répondent à des demandes qui voient dans la communication un moyen susceptible de garantir la cohésion entre les différents acteurs. Dans ces organisations, on compte sur les « spécialistes » de la communication pour garantir la paix sociale et minimiser la violence des nombreux conflits qui les traversent.

\section{Les réponses à la demande sociale}

19 Les étudiants en communication s'investissent dans la mission de comprendre le fonctionnement de la communication au sein des organisations. Toutefois, ils s'intéressent à l'analyse d'une question qui rend compte d'un ou plusieurs aspects de la communication organisationnelle sans pour autant l'extraire du contexte dans lequel elle s'intègre. Afin d'interroger et de comprendre le réel mais aussi de répondre à la demande sociale, ces futurs chargés de communication s'appuient sur la littérature abondante des Sic et proposent des modèles d'analyse qui s'en inspirent. L'objectif étant d'aider les organisations à améliorer les outils, canaux et stratégies de communication afin de mieux communiquer aussi bien en interne et qu'en externe.

\section{Analyses globales de la communication}

Dans leurs contacts avec les organisations, les futurs chargés de communication explorent diverses pistes afin de répondre à la demande sociale. Ils interrogent le fonctionnement organisationnel dans une perspective de résolution des problèmes liés à la communication. Pour ce faire, on mène deux grands types d'analyse, au fond très liés, de la communication. Le premier propose une analyse générale de la communication des organisations tandis que le second prend pour point de départ une question particulière, 
à partir de laquelle on tend éventuellement à comprendre le fonctionnement global de l'organisation.

La première piste de recherche est majoritaire. Elle se penche sur une analyse globale de la communication des organisations dans l'objectif affiché d'y apporter une amélioration. Après une période d'imprégnation de l'environnement de l'organisation, on entreprend un diagnostic de la communication visant l'impulsion de nouvelles formes de gestion. Les questions soulevées ont trait à la politique générale de communication organisationnelle et les objectifs sont divers. Il est souvent question de mettre en place un plan de communication dans des structures qui n'en disposaient pas auparavant. Les ambitions, impulsées parfois par les professionnels en place, ne se limitent pas à la mise en place d'un nouveau management et de nouvelles formes de gestion du personnel. Elles concernent également des domaines qui relèvent de questions précises: recherche des dysfonctionnements ou défaillances de la communication et réflexion sur les circonstances du changement en terme de résistance et de climat social. Dans le cadre d'une démarche de communication au service du management, l'audit vise des transformations profondes dans l'approche organisationnelle pour espérer favoriser le travail en commun, faire en sorte que les employés s'approprient les objectifs de l'organisation, favoriser la cohésion sociale, s'engager dans une démarche de qualité, soigner l'image et développer une culture d'entreprise.

La deuxième piste explorée par les futurs chargés de communication consiste en l'analyse d'un aspect particulier de la communication. Il s'agit d'une approche qui tente d'expliquer puis de fournir une réponse à un problème précis soulevé par les professionnels. Dès lors, on analyse la place d'un projet ou d'un service dans l'organisation générale. L'objectif visé consiste à étudier l'image d'un service auprès des autres services, à mesurer l'impact de l'activité d'un service au sein d'une structure dans le cas d'une institution publique. Il est également question de penser les implications d'une nouvelle orientation de gestion sur l'ensemble des composantes d'une entreprise. Il s'agit enfin de dynamiser une ou des activités d'un service.

\section{Quatre modèles de communication organisationnelle}

La formation en communication dans les DFA dispense différents courants et approches des mondes organisationnels. Afin de mener leurs travaux, les étudiants adoptent ces modèles d'analyse issus de la littérature existante en Sic. A la richesse des expériences menées sur le terrain répond une diversité de choix des approches d'analyse. Il n'en demeure pas moins que ces orientations peuvent être classées en quatre modèles.

Dans ce sens, on présente les approches de communication organisationnelle sur lesquelles reposent les grilles de lectures des étudiants en communication. Il primordial par ailleurs de reconnaître une démarche de combinaison de plusieurs approches pour résoudre les problématiques traitées et de leur adaptation parfois aux contextes historique, géographique et social des DFA.

En dépit de l'existence de divers modèles (psychologie des organisations, communication publique), on se cantonne pour l'essentiel à ceux rencontrés fréquemment lors de notre étude. Après une définition sommaire de ces modèles, on reviendra sur les contextes et circonstances de leur adaptation. 

stratégique. Cette dernière s'est imposée grâce aux travaux de Michel Crozier et Ehrard Friedberg ${ }^{10}$. Le modèle socio-technique, on le rappelle, est principalement orienté sur les « acteurs ». Ces derniers, avec leur liberté et leur rationalité limitée, tentent d'atteindre leurs propres objectifs (distincts des contraintes de l'organisation). Il existe autant d'acteurs que de stratégies car chacun fait et défait des alliances temporaires. Le but étant d'accroître son pouvoir. C'est là en effet un élément clef du modèle stratégique qui va permettre ensuite de considérer le pouvoir comme central et objet de toutes négociations et échanges entre acteurs ou groupes d'acteurs. On distingue quatre sources de pouvoir au sein de l'organisation : expertise et compétences difficilement remplaçables ; maîtrise des relations de l'organisation avec son environnement; la capacité à faire circuler de l'information; l'utilisation des règles organisationnelles. Le fonctionnement de l'organisation, par conséquence, découle d'un équilibre toujours altérable entre toutes ces stratégies opposées (Bouvier, 2004, 25-26). Dans ce système, marqué par les enjeux des acteurs, avec tout ce que cela comporte comme zones d'incertitudes, la régulation se fait en fonction des opérations internes conflictuelles. "L'analyse stratégique introduit de la sorte une nouvelle vue métaphorique des organisations, comme systèmes faits de coalitions changeantes et de négociations permanentes entre les acteurs et les soussystèmes $»^{11}$.

L'adaptation de l'approche stratégique par les étudiants en communication révèle des usages multiples. Elle sert le plus souvent de point de départ pour la compréhension du fonctionnement global des organisations et de leurs communications. Elle est utilisée également comme modèle d'analyse des rapports entre un service, notamment de communication, et l'ensemble de composantes de l'organisation. L'objectif est de déceler les problèmes qui entravent l'action de communication, de comprendre les freins au changement, de déterminer les motivations des acteurs en rapport avec la problématique étudiée, de proposer une stratégie efficace de communication.

\section{L'approche culturelle}

Sous l'influence de l'ethnologie et de l'anthropologie sociale, inspirée entre autres des travaux de Claude Lévi-Strauss, on commence à évoquer une culture d'entreprise notamment, en France, par Alain Touraine et Renaud Sainsaulieu. On s'accorde à dire qu'il existe des rapports étroits entre la culture de l'organisation et ses performances. Cette approche renvoie au cadre symbolique et des valeurs aussi bien explicites qu'implicites (Bouvier, 2004, 27-29). Porteuse de normes et rites, elle est la pièce angulaire du patrimoine de l'organisation. La culture est un construit collectif qui n'est pas figé certes, mais dont l'évolution est lente. Elle peut être par ailleurs un obstacle aux innovations et aux changements. La culture d'entreprise participe à la construction du sens collectif de l'action et s'insère dans un processus de socialisation. Elle est à l'origine de représentations susceptibles de créer une identité sociale favorisant le partage entre individus appartenant à l'organisation et facilitant ainsi la communication entre eux (Sainsaulieu, 1992). Cependant, les conflits internes ont pour origine les rapports entre les microcultures internes, différenciatrices des individus et groupes, et les macrocultures générales de l'organisation, unificatrices des ses acteurs.

C'est dans ce sens que cette approche intéresse les étudiants en communication qui considèrent l'organisation, entre autres, comme un microcosme social. Cette dernière est 
confrontée en effet à la complexité de cohabitation entre individus, aux aléas des rapports humains notamment de jalousie, de rivalités entre services. Existent également des problématiques de «couleur» ou de « race» relatives notamment aux DFA et sans lesquelles aucune compréhension des rapports au sein de l'organisation n'est saisie dans sa totale complexité (Daghmi, 2006). Les futurs chargés de communication adoptent d'autres grilles de lecture plaçant ainsi la communication au service du management. La communication interne est une réponse contre le cloisonnement des secteurs d'activités. Elle facilite le transfert d'expériences et renforce la cohérence et la réactivité des organisations (D'Almeida, Libaert, 2004, 28). Elle favorise les échanges de connaissances, d'expériences afin de faire partager et développer le progrès. C'est dans ce sens que la communication interne peut être considérée comme le moyen de développer la cohésion entre les employés de l'organisation et leur donner le sens du collectif (Detrié, MeslinBroyez, 2001).

Cette approche est adoptée afin de rendre compte du rôle du service de communication dans une organisation. Elle est aussi un moyen de mettre en place un plan ou une nouvelle politique de communication, de donner au management les moyens d'accompagner le changement. On cherche ainsi à faire adopter les objectifs de l'entreprise par les employés, à favoriser le travail en commun, à créer la cohésion entre les acteurs au sein de l'organisation.

\section{L'approche systémique}

31 Le paradigme de la systémique est fondé sur le principe qui tente de tenir compte de la complexité des objets étudiés en conciliant les liens entre le tout et les parties. On donne une importance capitale aux interactions au sein du système. Ce dernier est toujours pourvu d'un sous-système, de pilotage, dont l'objectif est de le réajuster et de le diriger suivant ses finalités. Il est question d'un effort permanent reposant sur les métacommunications et la volonté collective (Mucchielli, 1998, 111). Les conséquences d'un changement local sont difficilement prévisibles. Par conséquent, cette approche incite à admettre l'incertitude et à prendre en compte les parties et le tout lorsqu'on entreprend un changement. Son intérêt réside dans le rôle fondamental qu'elle donne aux interactions, au sein du système et en rapport avec son écosystème. Ainsi, la notion de sens prend naissance dans un contexte généralisable. Le contexte permet de forger la signification des échanges et, contextes et significations, se construisent grâce aux échanges eux-mêmes (Mucchielli, 2001, 96-97).

Le recours à l'analyse systémique, ou combinée à d'autres modèles, aide les étudiants en communication à comprendre les relations qu'entretient le service de communication avec les autres services. L'objectif est de mettre en place un plan de communication suite à un audit, d'analyser la conduite d'un changement ou d'améliorer la communication interne au sein de l'organisation.

\section{Réponses aux besoins stratégiques identifiés}

Ces études sont un révélateur de la nature des problèmes auxquels sont confrontées les organisations dans les DFA. Elles identifient les dysfonctionnements au niveau de la communication organisationnelle dont ces structures devront faire face. Les propositions révèlent avant tout, de manière claire et précise, le niveau de l'implantation de la 
communication dans ces organisations. Elles indiquent l'intérêt qu'on alloue et les représentations qu'on se fait du rôle et de la fonction de communication. Les organisations sont en réalité handicapées par l'ambiguïté ou le défaut de transparence quant à leurs projets d'entreprise, par l'insuffisance ou l'inadaptation des supports de communication et par les représentations de la fonction de communication.

Suite aux analyses menées à partir de thématiques en rapport avec la communication, les étudiants tentent de trouver des solutions susceptibles de répondre aux attentes des professionnels. Ainsi, les recommandations faites, à travers les analyses du terrain, concernent plusieurs niveaux.

Le premier type de préconisations porte sur des besoins stratégiques. Elle concerne la mise en place ou l'ajustement de plan de communication qui va bien au-delà d'une vision qui réduit la communication à une simple diffusion de l'information ou multiplication de supports. En effet, le plan de communication doit répondre à divers critères de souplesse, d'insertion dans la politique générale de l'organisation, d'adaptation aux besoins du moment et de prise en compte des relations au travail. Il doit faciliter une implication de l'ensemble des salariés par une politique d'accompagnement du management qui expose les alternatives et contraintes de l'organisation. La communication interne est tout un « dispositif et plan de communication, déroulant, dans le temps et dans l'espace des actions d'échanges »entre les acteurs de l'organisation et dirigé par sa hiérarchie (Mucchielli, 2001, 8). Le renforcement d'une communication verticale a non seulement des implications sur la réalisation du travail mais il influence aussi l'image de l'organisation et le climat social (Petit, Dubois, 1998, 38).

En définitive, le plan de communication permettra au service, et à la fonction de communication de sortir d'une dimension essentiellement technique qui la confine à un rôle de création et d'amélioration de supports de communication. Une telle représentation « managériale », force est de constater, est largement répandue dans les organisations des DFA (Daghmi, 2006).

Le deuxième type de propositions tourne autour des supports de communication. Les organisations souffrent de l'insuffisance ou de l'inadaptation des outils de communication. Dans certains cas, par manque de moyens ou de pouvoir et donc d'information, les services de communication peinent à créer ou à transformer les supports existants. La mise en place ou l'ajustement de ces derniers ne fait que rarement l'objet d'une évaluation auprès des utilisateurs (en terme d'usage, de satisfaction, de notoriété, etc.). En conséquence, ils ne remplissent que peu leurs fonctions de projets de diffusion d'informations servant à augmenter les connaissances globales autour de l'organisation.

Le troisième type de solutions concerne les rapports entre communication et management, autrement dit les moyens d'accompagnement de changement dans les organisations. Il s'agit de pistes d'ouverture sur une politique de communication qui guide et délivre au management les outils nécessaires au pilotage et à la maitrise du changement. Elles tournent autour de stratégies de communication faisant en sorte que les salariés s'approprient les nouveaux objectifs de l'organisation. Concrètement, il peut être question de rechercher l'amélioration de l'image d'un service ou d'une activité. Globalement, les solutions de management participatif visent à prendre davantage en compte et à comprendre les attentes et valeurs personnelles des salariés pour espérer améliorer le degré de leur motivation et implication (Plane, 2003, 37). 
39 Le quatrième type de préconisation met en valeur la culture de l'entreprise. Il s'agit essentiellement de structures en situation conflictuelle, avec des composantes éclatées géographiquement, des activités diverses ou subissant de grandes transitions. Elles ne présentent guère une identité unifiée de nature à favoriser des actions fédératrices. Dès lors, on accorde un rôle primordial au service ou à la politique de communication afin de mettre en commun un langage, des comportements, des valeurs, une culture dans le but de développer une appartenance à l'organisation.

Enfin, les solutions proposées à travers les études de terrain révèlent également un déficit au niveau des échanges et des réunions des salariés ou d'ignorance du rôle de la communication. Cela se traduit par le manque d'information de ces derniers et de surcroît d'implication dans la réalisation des objectifs de l'entreprise. Afin de mieux informer, les propositions tournent autour de rencontres thématiques, de réunionsdébats, de séminaires, de conférences, etc.

Formation et mondes professionnels : une rencontre délicate

L'enseignement des approches des mondes organisationnels à travers les concepts et théories de la communication prépare les étudiants à cerner les besoins de l'organisation en termes de communication. Il leur permet de saisir ce qui se joue dans ces organisations, d'en comprendre globalement les enjeux sociaux et, plus particulièrement, ceux liés aux contextes historique, géographique et socioéconomique.

La confrontation au terrain se révèle difficile en raison de la perception de la fonction de communication et de ses professionnels. La difficulté est accentuée également par le regard et la méfiance des réflexions qui découlent des théories et méthodes d'analyse des $\mathrm{Sic}^{12}$. Ces dernières, tout comme la fonction de communication, semblent avoir un long chemin à parcourir avant de gagner la reconnaissance des professionnels.

Ces préconisations faites dans le cadre de stage de formation correspondent à des cas concrets d'organisation, dans les DFA certes. Mais elles peuvent apporter des réponses, parfois générales, en mesure d'intéresser d'autres organisations et donner ainsi des idées aux étudiants qui seront confrontés à des cas similaires.

Cette réflexion est moins la description d'une quelconque spécificité des organisations des DFA qu'une approche générale sur des rapports entre deux mondes parfois très éloignés : formation universitaire et monde professionnel. La première développe des théories en rapport avec le terrain certes, mais dont l'intérêt varie selon les contextes et l'adoption suscite parfois la méfiance. Le second, même demandeur et ouvert sur le changement, doute de l'apport d'une discipline peu reconnue,paradoxalement et simultanément jugée nécessaire et peu crédible (Devèze, 1998, 37). La rencontre entre ces deux mondes, entre autres, à travers les étudiants en communication, reste aléatoire. Elle est dépendante d'un ensemble de paramètres loin d'être maîtrisables par ces étudiants.

En définitive, l'adoption des théories et pratiques universitaires des Sic par des étudiants en manque d'expérience dans le domaine de la communication révèle un aspect qui mérite l'approfondissement. Seuls et souvent diminués face à la complexité de l'organisation, certains étudiants sont handicapés par cet isolement face aux mondes organisationnels. A la difficulté ordinaire d'un stage s'ajoute celle du besoin de montrer l'utilité de la formation en communication, l'importance de la fonction de communication, au sens qu'on lui donne à l'université, et l'efficacité des méthodes d'analyses qui découlent de ce champ de recherche. Les résultats, bien entendu, sont loin d'être toujours décevants. Des étudiants en communication arrivent à imposer le respect 
aux professionnels par leurs approches et par la finesse de leurs analyses de la communication organisationnelle.

\section{BIBLIOGRAPHIE}

Bernoux P., Sociologie du changement dans les entreprises et les organisations, Paris, Seuil, 2004.

Bouvier A., Management et sciences cognitives, Paris, PUF, coll. «Que sais-je », 2004.

Daghmi F., "Sic et organisations en Martinique ». in: Questionner les pratiques d'information et de communication. Actes du XV Congrès de la Sfsic, Bordeaux, 2006, pp. 195-201.

Daghmi F., "Journalistes et identité en Martinique ». in: Communication et langages, $n^{\circ} 148$, juin 2006, pp. 103- 116

Davallon J., "Objet concret, objet scientifique, objet de recherche ». in : Hermès. $n^{\circ} 38.2004$, pp. 30-37.

Delcambre P. (dir.), Communications organisationnelles. Objets, pratiques, dispositifs, Rennes, Presse universitaire de Rennes, 2000.

Floris B., «Les ingénieurs de sens ». in : Sciences de la société. n 32, 1994, pp. 53-69.

Floris B., La communication managériale, la modernisation symbolique des entreprises, Grenoble, PUG, coll. «Communication, médias et sociétés », 1996.

Jeanneret Y., Ollivier B. (dir.), Les sciences de l'information et de la communication : savoirs et pouvoirs. Hermès, $n^{\circ}$ 38, CNRS, 2004.

La France et ses Outre-mers. L'enjeu du multiculturel. Hermès, n 32-33, CNRS, 2002.

Le Moënne C. (dir.), Communication des entreprises et des organisations, Rennes, Presse universitaire de Rennes, 1998.

Mucchielli A., Approche systémique et communicationnelle des organisations, Paris, Armand Colin, 1998.

Mucchielli A., La communication interne. Les clés d'un renouvellement, Paris, Armand Colin, 2001.

Mucchielli A., Les sciences de l'information et de la communication, Paris, Hachette, 2001.

Petit F., Dubois M., Introduction à la psychologie des organisations, Paris, Dunod, 1998.

Plane J-M., Théorie des organisations, Paris, Dunod, 2003.

Sainsaulieu R., L'entreprise une affaire de société, Paris, Presse de Sciences politiques, 1992.

\section{NOTES}

1. Avec la mise en place de la réforme LMD, l'UAG propose un Parcours Information, Communication et Ingénierie documentaire dans le cadre d'une nouvelle licence du domaine Lettres et Sciences humaines, mention Sciences du Langage, de la Communication et de la Société. Les modules de communication mis en place devraient préparer les étudiants à la fois à 
l'entrée en licence professionnelle de communication ou bien à la poursuite de leurs cursus en Master de communication prévu par l'établissement.

2. Dossier de renouvellement d'habilitation de la Licence professionnelle de communication, septembre 2005.

3. La formation a pour objectif de répondre à la demande locale en la matière. Le caractère national de la qualification obtenue n'est pas contradictoire avec une insertion professionnelle des diplômés majoritairement dans leurs régions d'origine.

4. Il s'agit de l'analyse du fonctionnement et des besoins des organisations, écoute active des interlocuteurs, conception/réalisation de stratégies de communication en utilisant différents supports (vidéo, PAO, Web, etc.), l'organisation d'événements, etc.

5. Les étudiants réalisent deux grandes actions de communication au cours de leur formation: analyse d'une commande d'organisation sur la communication externe et qui se concrétise par la mise en place de supports, l'organisation d'événements, etc. ; étude d'un ou de plusieurs aspects de la communication interne (stage de trois mois).

6. On dispense un savoir global, entre autres, dans le domaine des Sic. Les étudiants en communication adaptent ces théories et réflexions pratiques au terrain des organisations dans les DFA. Cela ne n'empêche pas le recours à d'autres appuis théoriques (sociologie, psychologie, management, etc.).

7. Ce travail qui découle de l'analyse des mémoires d'étudiants en communication réalisés lors de la formation pour l'obtention du diplôme de chargé de communication ainsi que d'une enquête auprès de ces mêmes étudiants. Après cinq années d'existence, et au moment où le ministère de l'Education nationale renouvelle son habilitation, la formation en communication s'interroge sur ses pratiques, sa formation, sa vocation, ses objectifs, ses méthodes d'enseignement et ses réponses à la demande organisationnelle dans les DFA.

8. Les organisations publiques (administrations, collectivités, etc.) sont le premier employeur des chargés de communication dans les DFA. Cela s'explique notamment par deux raisons : près de deux tiers du marché de l'emploi dans les DFA relèvent de l'Etat; la taille et l'importance des entreprises privées dans ces trois régions limitent le recours ou le recrutement de chargés de communication.

9. Les travaux de tous les étudiants sont fondés sur des enquêtes afin de confronter leurs problématiques au terrain. A répartition presque égale, ils ont recours au questionnaire, aux entretiens ou à la combinaison des deux.

10. Les travaux de Michel Crozier et de Ehrard Friedberg sont considérés comme la base de la sociologie des organisations française. L'œuvre classique des deux auteurs est: L'acteur et le système. Les contraintes de l'action collective. Paris, Seuil, 1977, 436 p.

11. Bouvier Alain, Management et sciences cognitives. Paris, PUF, coll. «Que sais-je », 2004, p. 26

12. Nos meilleurs étudiants, qui arrivent à adopter une approche critique correspondant aux exigences universitaires, qui cernent parfaitement la fonction de communication, les enjeux de pouvoir, les stratégies des acteurs, etc., au sein de l'organisation, peinent à se faire embaucher. Ce phénomène est accentué par l'étroitesse du marché dans les DFA. 


\section{RÉSUMÉS}

Les étudiants en communication s'investissent dans la mission de comprendre le fonctionnement de la communication au sein des organisations. Ils s'intéressent à l'analyse d'un ou plusieurs aspects de la communication organisationnelle sans pour autant l'extraire de son contexte. Afin d'interroger et de comprendre le réel mais aussi de répondre à la demande sociale, ces futurs chargés de communication s'appuient sur les Sic et proposent des modèles d'analyse qui s'en inspirent. L'objectif étant d'aider les organisations à améliorer les outils, canaux et stratégies de communication pour mieux communiquer aussi bien en interne qu'en externe.

The mission of students in communication is to understand the operation of communication within organizations. They are interested in the analysis of one or more aspects of organizational communication in context. In order to question and to understand the reality of organizations but also in order to satisfy their needs, these future communication professionals will use Information and Communication Science (Sic) and propose models of analysis which are based on same. The objective is to help the organizations to improve their tools, channels and strategies for better intra- and inter-organizational communication.

INDEX

Mots-clés : formation, Sic, chargés de communication, organisations, UAG, DFA

\section{AUTEUR}

\section{FATHALLAH DAGHMI}

Fathallah Daghmi est Maître de Conférences en SIC à l'Université de Poitiers, chercheur à Migrinter et associé au GREC/O. Ses travaux portent sur les interactions entre la formation en communication et le monde organisationnel. Après avoir étudié les représentations de l'immigration dans les médias français, il s'intéresse actuellement à l'identité en rapport avec les perceptions journalistiques et les traitements médiatiques. Mail : fdaghmi@iutang.univ-poitiers.fr 